\title{
Force Sensorless Fine Force Control Based on Notch-Type Friction-Free Disturbance Observers
}

\author{
Hiroshi Nakamura ${ }^{* a}$ \\ Yuki Yokokura* \\ Student Member, \\ Kiyoshi Ohishi* \\ Fellow \\ Toshimasa Miyazaki* Member, \\ Naoki Kamiya* \\ Student Member \\ Akifumi Tsukamoto** \\ Member
}

(Manuscript received April 7, 2017, revised July 28, 2017)

\begin{abstract}
In order to realize force sensorless fine force control, this paper proposes a new notch-type friction-free disturbance observer (DOB) and a new notch-type friction-free reaction force observer (FFRFO). Generally, a force sensorless force control system is always influenced by the static friction phenomenon. In order to suppress the estimation error due to static friction, a force sensorless force control system uses a dither signal, and a friction free reaction force observer (FFRFO) is used to consider this dither signal. Since the high frequency gain charactheristics of conventional FFRFO are significantly higher than $0 \mathrm{~dB}$, the entire force control system sometimes becomes unstable or produces oscillating responses. To overcome this problem, this paper proposes a new force sensorless control system using the notch-type friction free reaction force observer, whose inner system is the proposed acceleration control based on the notch-type friction free disturbance observer. The effectiveness of the proposed system is confirmed by experiments using an actual industrial robot.
\end{abstract}

Keywords: force sensorless force control, disturbance observer, reaction force observer, industrial robot

\section{Introduction}

Recently, robots have been employed during the manufacturing process in applications such as cutting, deburring, grinding, polishing, and assembling ${ }^{(1)}$. Force control is important to these tasks and extensive studies are being carried out in order to improve the performance of force servoing ${ }^{(2)(3)}$. In general, a force sensor is required for the detection of an external force and the construction of feedback control systems. In the reference papers ${ }^{(4)-(8)}$, built-in torque sensors has been used for controlling the contact force. Although precise force control can be achieved by using force sensors, their use often has several drawbacks such as low stiffness and increased cost.

Meanwhile, force sensorless force control using observer techniques has been studied in order to overcome the problem, with regard to cost efficiency. One of the conventional methods is the reaction force observer method, which is capable of estimating the external force by using a reference current and motor velocity ${ }^{(9)}$. However, estimation accuracy is affected negatively by nonlinear friction such as static friction. Generally, the force control for product assembly requires precision within $\pm 5 \mathrm{~N}$ for reference. The proposed force sensorless force control method satisfies this requirement, and considerably reduces the sensor cost. Therefore,

a) Correspondence to: Hiroshi Nakamura. E-mail: s133152@stn. nagaokaut.ac.jp

* Motion Control Labs., Nagaoka University of Technology 1603-1, Kamitomioka, Nagaoka, Niigata 940-2188, Japan

** Hirata Corporation

111, Hitotsugi, Ueki, Kita, Kumamoto 861-0198, Japan the force sensorless force control system is extremely effective for robot force control applications.

In order to solve the problem of static friction, the frictionfree disturbance observer (DOB) and the friction-free reaction force observer (FFRFO) have been proposed ${ }^{(10)-(13)}$. The dither signal is superimposed to the reference current so that the motor system will not operate mostly in the zero velocity region. As a result, the estimation performance of FFRFO is not affected by static friction.

Furthermore, this paper uses velocity-regulator-based force control in order to suppress mechanical vibration caused by reduction gearing systems. One of the vibration suppression techniques suitable to force control is resonance ratio control (RRC) ${ }^{(14)-(18)}$. The RRC appropriately changes the moment of motor-side inertia equivalently, so that the resonant vibration can be suppressed. However, RRC sometimes cannot suppress the resonant vibration when the bandwidth of the disturbance observer implementing RRC is lower than that of the resonant frequency. Since our experimental equipment has ball screw gears, the resonant vibration frequency is higher than the estimation cutoff frequency.

This paper proposes a new force sensorless force control system using the notch-type FFRFO, whose inner system is the proposed acceleration control based on the notch-type friction free DOB. In the case of conventional FFRFO, since the gain characteristics in high frequency regions are significantly increased with respect to $0 \mathrm{~dB}$, the whole force control system sometimes becomes unstable or the oscillated responses. To overcome this problem, this paper realizes a force sensorless force control system based on the notch-type friction-free DOB and FFRFO. 


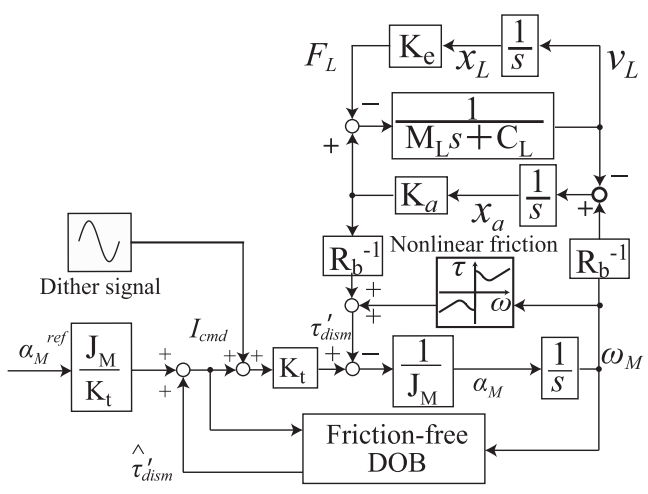

Fig. 1. Plant system and motor-side acceleration controller using friction-free DOB and dither signal

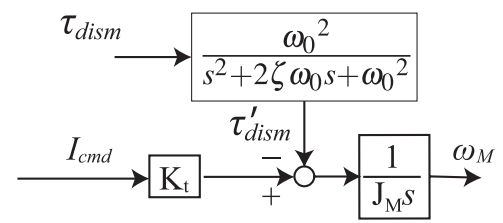

Fig. 2. Disturbance model for conventional and proposed friction-free DOBs

\section{Friction-free Disturbance Observer}

In this paper, the dither signal is used in order to reduce the effect of nonlinear static friction. A friction-free DOB is adopted for the purpose of preventing interference between the dither signal and the acceleration controller's disturbance compensation. Figure 1 shows the plant system and acceleration contoller based on the friction-free DOB. $J_{M}, K_{t}, R_{b}, K_{a}, M_{L}, C_{L}$, and $K_{e}$ denote the motor inertia, torque constant, gear ratio, stiffness, load-side mass, viscosity friction, and environmental stiffness, respectively. $I_{c m d}, \alpha_{M}, \omega_{M}, x_{a}, x_{L}, v_{L}$, and $F_{L}$ represent the current reference, motor acceleration, velocity, deformation, load-side position, velocity, and load force.

2.1 Zero-order Friction-free Disturbance Observer (Conventional Multiple Roots Design) The friction-free disturbance observer is designed by using the model shown in Fig. $2^{(13)}$. The motor disturbance torque $\tau_{\text {dism }}^{\prime}$ is modeled by the second-order system with dither angular frequency $\omega_{0}$ and damping factor $\zeta$. Therefore, the motor disturbance torque $\tau_{\text {dism }}^{\prime}$ is expressed as follows:

$$
\tau_{d i s m}^{\prime}=\frac{\omega_{0}^{2}}{s^{2}+2 \zeta \omega_{0} s+\omega_{0}^{2}} \tau_{d i s m}
$$

where $\tau_{\text {dism }}$ represents the disturbance torque removed the effect of the dither signal. The conventional zero-order frictionfree DOB treats $\tau_{d i s m}$ as the constant DC signal. The state equations of the zero-order friction-free DOB are expressed as follows:

$$
\begin{aligned}
\dot{\boldsymbol{x}}_{d 0} & =\boldsymbol{A}_{d 0} \boldsymbol{x}_{d 0}+\boldsymbol{b}_{d 0} u_{d 0}+\boldsymbol{k}_{d 0} y_{d 0} \cdots \\
u_{d 0} & =I_{c m d} \quad y_{d 0}=\omega_{M} \\
\boldsymbol{A}_{d 0} & =\left[\begin{array}{cccc}
-k_{d 01} & J_{M}^{-1} & 0 & 0 \\
-k_{d 02} & 0 & 1 & 0 \\
-k_{d 03} & 0 & 0 & 1 \\
-k_{d 04} & 0 & -\omega_{0}^{2} & -2 \zeta \omega_{0}
\end{array}\right]
\end{aligned}
$$

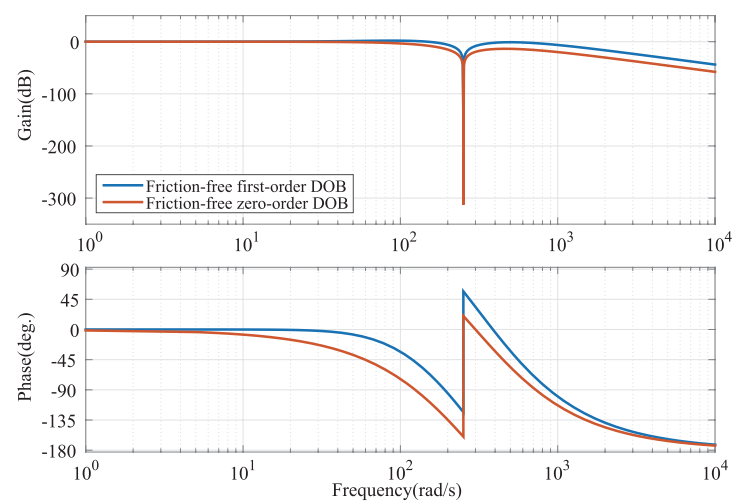

Fig. 3. Estimation characteristics of conventional zero and first order friction-free DOBs (multiple roots design) $\left(\hat{\tau}_{\text {dism }}^{\prime} / \tau_{\text {dism }}\right)$

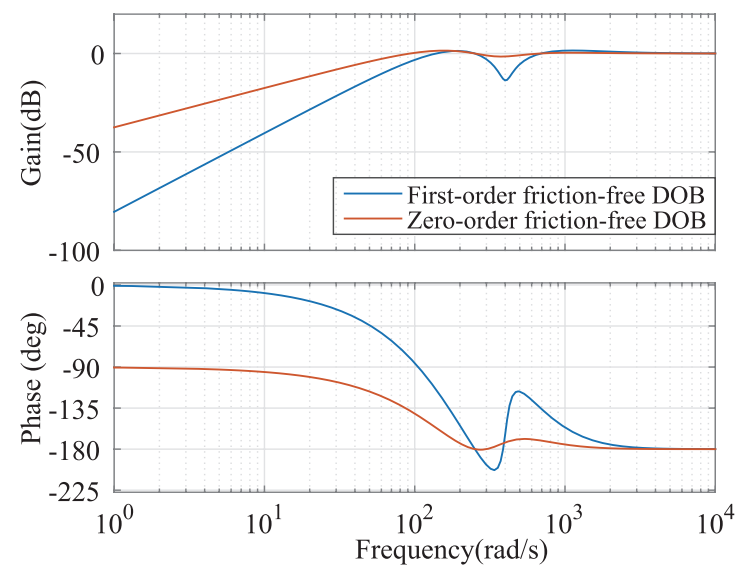

Fig. 4. Disturbance rejection responses of acceleration controllers using conventional zero and first order friction-free DOBs (multiple roots design) $\left(\tau_{\text {in }} / \tau_{\text {dism }}\right)$

$$
\begin{aligned}
& \boldsymbol{x}_{d 0}=\left[\begin{array}{llll}
\hat{\omega}_{M} & \hat{\tau}_{\text {dism }}^{\prime} & \hat{\boldsymbol{\tau}}_{\text {dism }}^{\prime} & \hat{\bar{\tau}}_{d i s m}^{\prime}
\end{array}\right]^{T} \\
& \boldsymbol{b}_{d 0}=\left[\begin{array}{llll}
K_{t} J_{M}^{-1} & 0 & 0 & 0
\end{array}\right]^{T} \\
& \boldsymbol{k}_{d 0}=\left[\begin{array}{llll}
k_{d 01} & k_{d 02} & k_{d 03} & k_{d 04}
\end{array}\right]^{T}
\end{aligned}
$$

where $\boldsymbol{k}_{d 0}$ is the gain vector of the zero-order friction-free DOB.

2.2 First-order Friction-free Disturbance Observer (Multiple Roots Design) In order to improve the disturbance suppression performance of the acceleration control in the low frequency band as shown in Fig. 3 and Fig. 4, this paper also designs the first-order friction-free DOB that treats $\tau_{\text {dism }}$ as the ramp signal. Therefore, the disturbance torque $\tau_{\text {dism }}$ is expressed as follows:

$$
\frac{d}{d t} \tau_{d i s m}=\dot{\tau}_{\text {dism }}, \frac{d}{d t} \dot{\tau}_{\text {dism }}=0 .
$$

Moreover, the disturbance torque $\tau_{\text {dism }}$ are expressed as follows from Eq. (1):

$$
\begin{aligned}
& \frac{d}{d t} \tau_{d i s m}^{\prime}=\dot{\tau}_{d i s m}^{\prime} \\
& \frac{d}{d t} \dot{\tau}_{d i s m}^{\prime}=\ddot{\tau}_{d i s m}^{\prime} \\
& \frac{d}{d t} \ddot{\tau}_{d i s m}^{\prime}=-\omega_{0}{ }^{2} \dot{\tau}_{d i s m}^{\prime}-2 \zeta \omega_{0} \ddot{\tau}_{d i s m}^{\prime}+\omega_{0}^{2} \dot{\tau}_{d i s m} . \cdots \cdots
\end{aligned}
$$

The state equations of the first-order friction-free DOB are expressed as follows from Fig. 2 and Eqs. (3)-(6): 


$$
\begin{aligned}
& \dot{\boldsymbol{x}}_{d 1}=\boldsymbol{A}_{d 1} \boldsymbol{x}_{d 1}+\boldsymbol{b}_{d 1} u_{d 1}+\boldsymbol{k}_{d 1} y_{d} \\
& u_{d 1}=I_{c m d} \quad y_{d 1}=\omega_{M} \\
& \boldsymbol{A}_{d 1}=\left[\begin{array}{ccccc}
-k_{d 11} & J_{M}^{-1} & 0 & 0 & 0 \\
-k_{d 12} & 0 & 1 & 0 & 0 \\
-k_{d 13} & 0 & 0 & 1 & 0 \\
-k_{d 14} & 0 & -\omega_{0}^{2} & -2 \zeta \omega_{0} & \omega_{0}^{2} \\
-k_{d 15} & 0 & 0 & 0 & 0
\end{array}\right]
\end{aligned}
$$

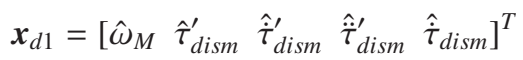

$$
\begin{aligned}
& \boldsymbol{b}_{d 1}=\left[\begin{array}{lllll}
K_{t} J_{M}^{-1} & 0 & 0 & 0 & 0
\end{array}\right]^{T} \\
& \boldsymbol{k}_{d 1}=\left[\begin{array}{lllll}
k_{d 11} & k_{d 12} & k_{d 13} & k_{d 14} & k_{d 15}
\end{array}\right]^{T}
\end{aligned}
$$

where $\boldsymbol{k}_{d 1}$ is the gain vector of the first-order friction-free DOB. When the observer poles $p_{d}$ are multiple root, the observer gains are expressed as follows:

$$
\begin{aligned}
& k_{d 11}=-5 p_{d}-2 \zeta \omega_{0} \\
& k_{d 12}=J_{M}\left(-10 p_{d}{ }^{2}-10 \zeta \omega_{0} p_{d}-4 \zeta^{2} \omega_{0}^{2}+\omega_{0}^{2}\right) \cdots \\
& k_{d 13}=J_{M}\left(10 p_{d}^{3}+20 \zeta \omega_{0} p_{d}^{2}+20 \zeta^{2} \omega_{0}^{2} p_{d}\right. \\
& \left.-5 \omega_{0}^{2} p_{d}+8 \zeta^{3} \omega_{0}^{3}-4 \zeta \omega_{0}^{3}\right) \\
& k_{d 14}=J_{M}\left(-5 p_{d}{ }^{4}-20 \zeta \omega_{0} p_{d}{ }^{3}+10 \omega_{0}{ }^{2} p_{d}{ }^{2}\right. \\
& -40 \zeta^{2} \omega_{0}^{2} p_{d}^{2}+20 \zeta \omega_{0}^{3} p_{d}-40 \zeta^{3} \omega_{0}^{3} p_{d} \\
& \left.+12 \zeta^{2} \omega_{0}^{4}-\omega_{0}^{4}-16 \zeta^{4} \omega_{0}^{4}\right) \\
& k_{d 15}=J_{M} \omega_{0}^{-2} p_{d}^{5}
\end{aligned}
$$

The estimated value of the disturbance torque $\hat{\tau}_{\text {dism }}$ is calculated using the estimated values of Eq. (7).

$$
\hat{\tau}_{d i s m}=\frac{\hat{\bar{\tau}}_{d i s m}^{\prime}+2 \zeta \omega_{0} \hat{\bar{\tau}}_{d i s m}^{\prime}+\omega_{0}^{2} \hat{\tau}_{d i s m}^{\prime}}{\omega_{0}^{2}} .
$$

Figure 3 shows the bode diagrams of both friction-free DOBs from the motor disturbance torque $\tau_{d i s m}^{\prime}$ to the actual disturbance torque $\hat{\tau}_{\text {dism }}$. The first-order friction-free DOB improves the gain attenuation and estimated delay in the low frequency band in comparison with the zero-order DOB.

Figure 4 shows the sensitivity bode diagrams against disturbance torque $\left(\tau_{\text {in }} / \tau_{\text {dism }}\right)$, of the acceleration control system. The disturbance suppression performance of the firstorder friction-free DOB is higher than that of the zero-order DOB.

2.3 Estimation Characteristics of Zero and Firstorder Friction-free DOB (Multiple Roots Design) In the case where poles of the zero-order friction-free DOB are designed with multiple roots, the estimation characteristic from motor disturbance $\tau_{\text {dism }}^{\prime}$ to the disturbance torque removed the effect of the dither signal $\tau_{d i s m}$ is expressed as follows:

$$
L_{d 0 m}(s)=\frac{\hat{\tau}_{d i s m}}{\tau_{d i s m}^{\prime}}=\frac{s^{2}+2 \zeta \omega_{0} s+\omega_{0}^{2}}{\omega_{0}^{2}} \frac{p_{d}^{4}}{\left(s-p_{d}\right)^{4}} .
$$

Similarly, the estimation characteristics of the first-order friction-free DOB are expressed as follows:

$$
L_{d 1 m}(s)=\frac{\hat{\tau}_{d i s m}}{\tau_{d i s m}^{\prime}}=\frac{s^{2}+2 \zeta \omega_{0} s+\omega_{0}^{2}}{\omega_{0}^{2}} \frac{p_{d}^{4}\left(5 s-p_{d}\right)}{\left(s-p_{d}\right)^{5}} .
$$

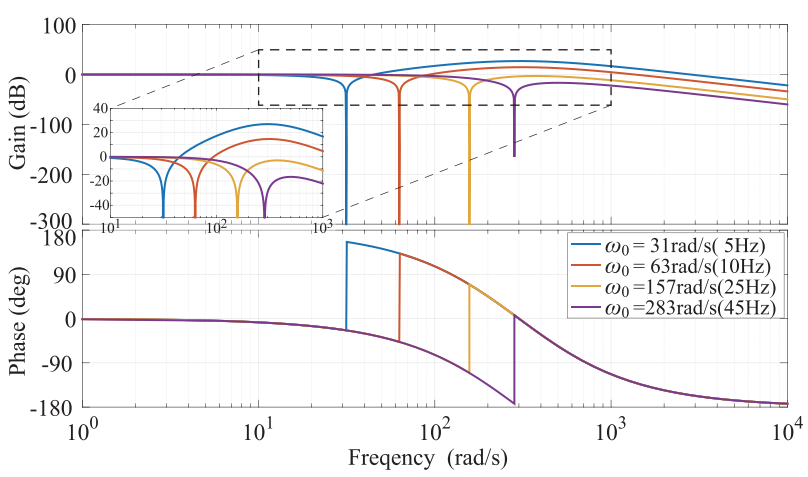

Fig. 5. Estimation characteristics of conventional zeroorder friction-free DOB when changing dither frequency $\omega_{0}$ (conventional multiple roots design)

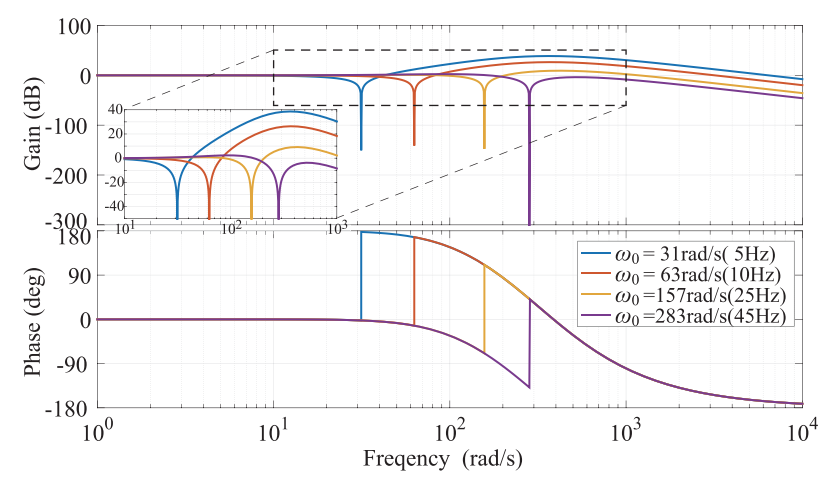

Fig. 6. Estimation characteristics of first-order frictionfree DOB when changing dither frequency $\omega_{0}$ (multiple roots design)

From Eq. (14) and Eq. (15) the estimation characteristics of friction-free DOB are shown as a cascade connection of the inverse second-order vibration model and a low-pass filter. Figures 5 and 6 show the estimation characteristics of the zero-order friction-free DOB and the first-order frictionfree DOB when changing the dither frequency $\omega_{0}$ from $10 \mathrm{~Hz}$ to $50 \mathrm{~Hz}$. In these figures, the poles of friction-free DOBs are set to $300 \mathrm{rad} / \mathrm{s}$. Since the gain characteristic of the inverse second-order vibration model increases as frequency increases, the robustness of the acceleration control system descends as dither signal frequency descends. Therefore, the dither frequency should be set to near the observer's poles, when the observer's poles are set to multiple roots.

2.4 Proposed Friction-free Disturbance Observer (Notch-type Design) In the case where the observer's poles are set to multiple roots, the dither frequency is limited from the view point of robust stability. The cause of this problem is that the inverse second-order vibration model is non-proper in Eq. (14) and Eq. (15). In this paper, the poles of friction-free DOB are redesigned so that the vibration model of estimation characteristics is proper. Considering the vibration model's numerator, the denominator of the zero-order friction-free DOB is redesigned as follows:

$$
\begin{aligned}
& \left(s^{2}+2 \zeta_{2} \omega_{0} s+\omega_{0}^{2}\right)\left(s-p_{d}\right)^{2} \\
& \quad=s^{4}+d_{1 n 3} s^{3}+d_{1 n 2} s^{2}+d_{1 n 1} s+d_{1 n 0} \\
& d_{0 n 3}=2 \zeta_{2}-2 p_{d} \\
& d_{0 n 2}=p_{d}^{2}-4 p_{d} \zeta_{2}+\omega_{0}^{2} \\
& d_{0 n 1}=2 p_{d}^{2} \zeta_{2}-2 p_{d} \omega_{0}^{2}
\end{aligned}
$$




$$
d_{0 n 0}=\omega_{0} 2 p_{d}^{2} .
$$

From Eq. (16) and Eq. (2), the observer gains of the notchtype zero-order friction-free DOB are expressed as follows:

$$
\begin{aligned}
& k_{d 0 n 1}=2 \zeta_{2}-2 p_{d}-2 \zeta_{1} \omega_{0} \\
& k_{d 0 n 2}=-4 J_{M} \zeta_{1}^{2} \omega_{0}^{2}+4 J_{M} \zeta_{1} \omega_{0}\left(\zeta_{2}-p_{d}\right) \\
& +J_{M} p_{d}\left(4 \zeta_{2}-p_{d}\right) \\
& k_{d 0 n 3}=2 J_{M}\left\{\zeta_{1} \omega_{0}^{3}\left(4 \zeta_{1}^{2}-1\right)+\omega_{0}^{2}\left(4 \zeta_{1}^{2} p_{d}-4 \zeta_{1}^{2} \zeta_{2}+\zeta_{2}\right)\right. \\
& \left.+p_{d} \zeta_{1} \omega_{0}\left(p_{d}-4 \zeta_{2}\right)-\zeta_{2} p_{d}^{2}\right\} \\
& k_{d 0 n 4}=4 J_{M} \omega_{0}\left\{2 \zeta_{1}^{2} \omega_{0}^{3}\left(1-2 \zeta_{1}^{2}\right)+\zeta_{1} \omega_{0}^{2}\left(4 \zeta_{1}^{2} \zeta_{2}-4 \zeta_{1}^{2} p_{d}\right.\right. \\
& \left.\left.+p_{d}-2 \zeta_{2}\right)+p_{d} \omega_{0}\left(4 \zeta_{1}^{2} \zeta_{2}-\zeta_{1}^{2} p_{d}-\zeta_{2}\right)+\zeta_{1} \zeta_{2} p_{d}^{2}\right\}
\end{aligned}
$$

Similarly, the denominator and observer gains of first-order friction-free DOB are expressed as follows:

$$
\begin{aligned}
& \left(s^{2}+2 \zeta_{2} \omega_{0} s+\omega_{0}^{2}\right)\left(s-p_{d}\right)^{3} \\
& \quad=s^{5}+d_{1 n 4} s^{4}+d_{1 n 3} s^{3}+d_{1 n 2} s^{2}+d_{1 n 1} s+d_{1 n 0}
\end{aligned}
$$

$d_{1 n 4}=2 \zeta_{2} \omega_{0}-3 p_{d}$

$d_{1 n 3}=3 p_{d}^{2}-6 p_{d} \zeta_{2} \omega_{0}+\omega_{0}^{2}$

$d_{1 n 2}=6 p_{d}^{2} \zeta_{2} \omega_{0}-p_{d}^{3}-3 p_{d} \omega_{0}^{2}$

$d_{1 n 1}=3 p_{d}^{2} \omega_{0}^{2}-2 p_{d}^{3} \zeta_{2} \omega_{0}$

$d_{1 n 0}=-\omega_{0}^{2} p_{d}^{3}$.

$$
k_{d 1 n 1}=2 \omega_{0}\left(\zeta_{2}-\zeta_{1}\right)-3 p_{d}
$$$$
k_{d 1 n 2}=2 J_{M} \omega_{0}\left(\zeta_{2}-\zeta_{1}\right)\left(2 \zeta_{1} \omega_{0}+3 p_{d}\right)-3 J_{M} p_{d}^{2}
$$

$$
k_{d 1 n 3}=2 J_{M} \omega_{0}\left(\zeta_{2}-\zeta_{1}\right)\left\{\left(1-4 \zeta_{1}^{2}\right) \omega_{0}^{2}-6 \zeta_{1} \omega_{0}-3 p_{d}^{2}\right\}
$$

$$
+J_{M} p_{d}^{3}
$$

$$
k_{d 1 n 4}=2 J_{M} \omega_{0}\left(\zeta_{2}-\zeta_{1}\right)\left\{4 \zeta_{1} \omega_{0}^{3}\left(2 \zeta_{1}^{2}-1\right)+3 p_{d} \omega_{0}^{2}\left(4 \zeta_{1}^{2}-1\right)\right.
$$$$
\left.+6 p_{d}^{2} \zeta_{1} \omega_{0}+p_{d}^{3}\right\}
$$

$$
k_{d 1 n 5}=J_{M} p_{d}^{3}
$$

As a result, the estimation characteristics of notch-type zeroorder friction-free DOB are expressed as follows:

$$
L_{d 0 n}(s)=\frac{\hat{\tau}_{d i s m}}{\tau_{d i s m}^{\prime}}=\frac{s^{2}+2 \zeta_{1} \omega_{0} s+\omega_{0}^{2}}{s^{2}+2 \zeta_{2} \omega_{0} s+\omega_{0}^{2}} \frac{p_{d}^{2}}{\left(s-p_{d}\right)^{2}} .
$$

Similarly, the estimation characteristics of notch-type firstorder friction-free DOB are expressed as follows:

$$
\begin{aligned}
L_{d 1 n}(s)= & \frac{\hat{\tau}_{d i s m}}{\tau_{d i s m}^{\prime}}=\frac{s^{2}+2 \zeta_{1} \omega_{0} s+\omega_{0}^{2}}{s^{2}+2 \zeta_{2} \omega_{0} s+\omega_{0}^{2}} \\
& \frac{p_{d}^{2}\left\{\left(3 \omega_{0}-2 p_{d} \zeta_{2}\right) s-p_{d} \omega_{0}\right\}}{\omega_{0}\left(s-p_{d}\right)^{3}} .
\end{aligned}
$$

This paper calls this redesigned friction-free DOB the notchtype friction-free DOB. Figures 7 and 8 show the estimation characteristics of these notch-type friction-free DOBs. The gain characteristics shown in Figs. 7 and 8 in high frequency regions are lower than the characteristics shown in Figs. 5 and 6.

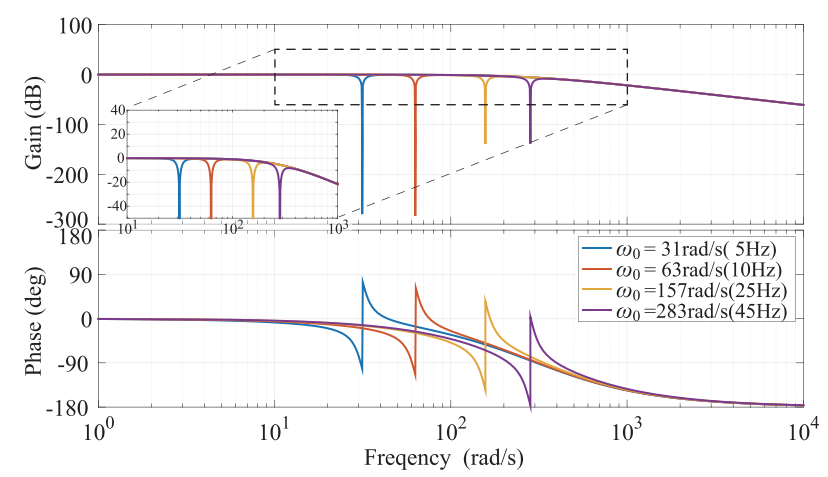

Fig. 7. Estimation characteristics of zero-order frictionfree DOB when changing dither frequency $\omega_{0}$ (proposed notch-type design)

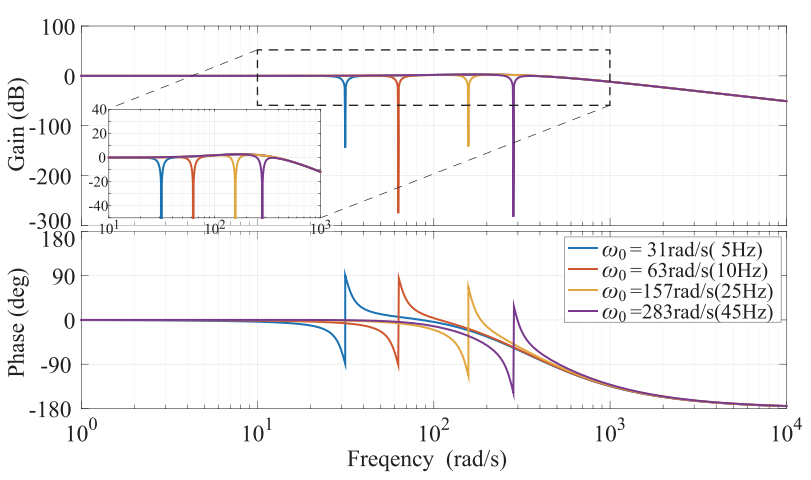

Fig. 8. Estimation characteristics of first-order frictionfree DOB when changing dither frequency $\omega_{0}$ (proposed notch-type design)

\section{Friction-free Reaction Force Observer (FFRFO)}

\subsection{Conventional FFRFO (Multiple Roots Design)} Similar to motor-side disturbance, the load-side force $F_{L}$ is vibrated by the dither signal effect. Therefore, it is necessary to remove the dither signal effect from the estimated loadside force. The robot arms are modeled as a two-inertia system. Figure 9 shows the plant model for the case where the dither signal is superimposed on the current command $I_{c m d}$. $A$ and $F_{s}$ represent the amplitude of the dither signal and deformation force. The external force $F_{\text {ext }}$ is transferred to the load-side disturbance $F_{L}$ which has the same frequency as the dither signal. Therefore, the load-side disturbance force $F_{L}$ is expressed using the external force $F_{\text {ext }}$ as follows:

$$
F_{L}=\frac{\omega_{0}^{2}}{s^{2}+2 \zeta \omega_{0} s+\omega_{0}^{2}} F_{e x t} .
$$

where $\omega_{0}$ represents the angular frequency of the dither signal and $\zeta$ denotes the damping coefficient. The FFRFO is designed using the model shown in Fig. 9. The state equation of the FFRFO are expressed as follows:

$$
\begin{aligned}
\dot{\mathbf{x}}_{r}(t) & =\mathbf{A}_{r} \mathbf{x}_{r}(t)+\mathbf{b}_{r} u_{r}(t) \\
\mathbf{y}_{r}(t) & =\mathbf{C}_{r} \mathbf{x}_{r}(t) \cdots \cdots
\end{aligned}
$$




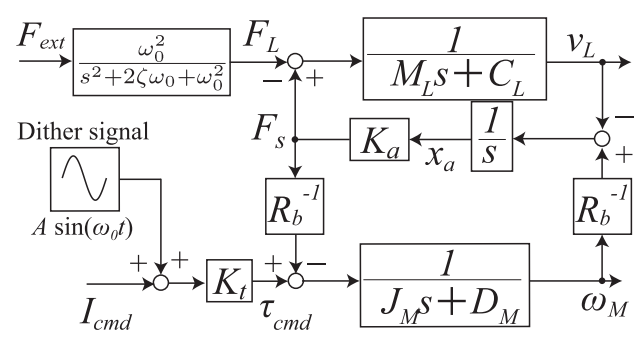

Fig. 9. Two-inertia system considering dither signal

$$
\begin{aligned}
& \mathbf{A}_{r}=\left[\begin{array}{cccccc}
a_{11} & a_{12} & 0 & 0 & 0 & 0 \\
a_{21} & 0 & a_{23} & 0 & 0 & 0 \\
a_{31} & a_{32} & a_{33} & a_{34} & 0 & 0 \\
a_{41} & 0 & 0 & 0 & a_{45} & 0 \\
a_{51} & 0 & 0 & 0 & 0 & a_{56} \\
a_{61} & 0 & 0 & 0 & a_{65} & a_{66}
\end{array}\right] \\
& \mathbf{b}_{r}=\left[\begin{array}{cc}
k_{r 1} & \frac{K_{t}}{J_{M}} \\
k_{r 2} & 0 \\
k_{r 3} & 0 \\
k_{r 4} & 0 \\
k_{r 5} & 0 \\
k_{r 6} & 0
\end{array}\right], \mathbf{C}_{r}=\left[\begin{array}{cccccc}
1 & 0 & 0 & 0 & 0 & 0 \\
0 & 1 & 0 & 0 & 0 & 0 \\
0 & 0 & 1 & 0 & 0 & 0 \\
0 & 0 & 0 & 1 & 0 & 0 \\
0 & 0 & 0 & 0 & 1 & 0 \\
0 & 0 & 0 & 0 & 0 & 1
\end{array}\right] \\
& \mathbf{x}_{r}(t)=\left[\omega_{M}(t) x_{a}(t) v_{L}(t) F_{L}(t) \dot{F}_{L}(t) \ddot{F}_{L}(t)\right]^{T} \text {, } \\
& u_{r}(t)=\left[\begin{array}{cc}
\omega_{M}(t) & I_{c m d}(t)
\end{array}\right]^{T} \\
& a_{11}=-\frac{D_{M}}{J_{M}}-k_{r 1}, \quad a_{12}=-\frac{K_{a}}{J_{M} R_{b}}, \\
& a_{21}=\frac{1}{R_{b}}-k_{r 2}, \quad a_{23}=-1, \quad a_{31}=-k_{r 3}, \\
& a_{32}=\frac{K_{a}}{M_{L}}, a_{33}=-\frac{C_{L}}{M_{L}}, \quad a_{34}=-\frac{1}{M_{L}}, \\
& a_{41}=-k_{r 4}, a_{45}=1 \text {, } \\
& a_{51}=-k_{r 5}, a_{56}=1 \text {, } \\
& a_{61}=-k_{r 6}, \quad a_{65}=-\omega_{0}^{2}, \quad a_{66}=-2 \zeta \omega_{0}
\end{aligned}
$$

where $k_{r 1}, k_{r 2}, k_{r 3}, k_{r 4}, k_{r 5}$, and $k_{r 6}$ denote the observer gains of FFRFO. The estimation of the reaction force $\hat{F}_{\text {ext }}$ is calculated by using estimation values $\hat{F}_{L}, \hat{\dot{F}}_{L}$, and $\hat{\vec{F}}_{L}$ from the FFRFO as follows:

$$
\hat{F}_{\text {ext }}=\frac{\hat{\vec{F}}_{L}+2 \zeta \omega_{0} \hat{\dot{F}}_{L}+\omega_{0}^{2} \hat{F}_{L}}{\omega_{0}^{2}} .
$$

Thus, the estimation of the reaction force is achieved without the dither signal effect. The dither signal reduces the influence on the static friction from the estimation of the reaction force $\hat{F}_{\text {ext }}$.

3.2 Proposed FFRFO (Notch-type Design) In the case where the dither frequency is set to low, the estimated external force $\hat{F}_{L}$ is amplified in the frequency band by the dither frequency to the poles of the FFRFO. In this case, the estimated external force $\hat{F}_{L}$ is noisy. Hence, this paper proposes a notch-type FFRFO similar to the friction-free DOB. The denominator of the notch-type FFRFO is expressed as follows:

$$
\begin{aligned}
& \left(s^{2}+2 \zeta_{2} \omega_{0} s+\omega_{0}^{2}\right)\left(s-p_{r}\right)^{4} \\
& \quad=s^{5}+d_{r n 4} s^{4}+d_{r n 3} s^{3}+d_{r n 2} s^{2}+d_{r n 1} s+d_{r n 0}
\end{aligned}
$$

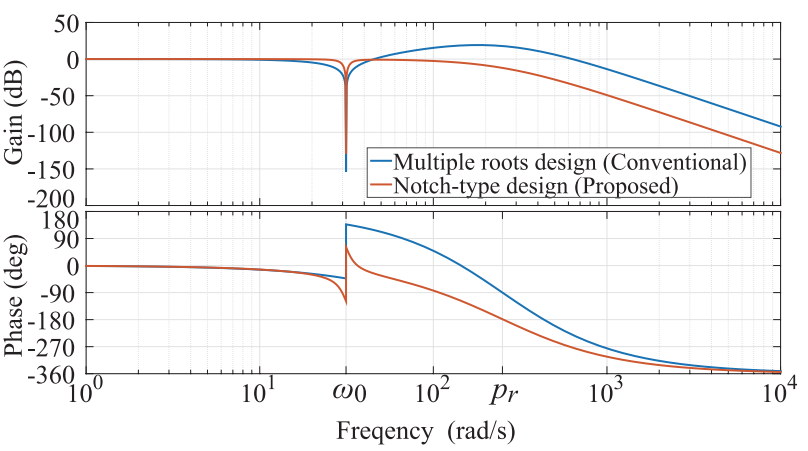

Fig. 10. Estimation characteristics of conventional and proposed FFRFOs from $F_{L}$ to $\hat{F}_{\text {ext }}$

$$
\begin{aligned}
& d_{r n 5}=-4 p_{r}+2 \zeta_{2} \omega_{0} \\
& d_{r n 4}=6 p_{r}^{2}-8 p_{r} \zeta_{2} \omega_{0}+\omega_{0}^{2} \\
& d_{r n 3}=-4 p_{r}^{3}+12 p_{r}^{2} \zeta_{2} \omega_{0}-4 p_{r} \omega_{0}^{2} \\
& d_{r n 2}=p_{r}^{4}-8 p_{r}^{3} \zeta_{2} \omega_{0}+6 p_{r}^{2} \omega_{0}^{2} \\
& d_{r n 1}=2 p_{r}^{4} \zeta_{2} \omega_{0}-4 p_{r}^{3} \omega_{0}^{2} \\
& d_{r n 0}=\omega_{0}^{2} p_{r}^{4} .
\end{aligned}
$$

From Eqs. (30), (31) and (33), observer gains of notch-type FFRFO are expressed as follows:

$$
\begin{aligned}
& k_{r 1}=2 \omega_{0}\left(\zeta_{2}-\zeta_{1}\right)-4 p_{r}-M_{L}^{-1} C_{L}-J_{M}^{-1} D_{M} \cdots \\
& k_{r 2}=J_{M} R_{b} K_{a}^{-1}\left\{4 \zeta_{1}\left(\zeta_{1}+\zeta_{2}\right) \omega_{0}^{2}\right. \\
& +2\left(\zeta_{2}-\zeta_{1}\right)\left(4 p_{r}+C_{L} M_{L}^{-1}\right) \omega_{0} \\
& \left.+\left(M_{L}^{-1} K_{a}-M_{L}^{-1} C_{L}^{2}-6 p_{r}^{2}-4 C_{L} p_{r}\right)\right\}+R_{b}^{-1} \\
& k_{r 3}=J_{M} R_{b} K_{a}^{-1}\left\{2\left(1-4 \zeta_{1}^{2}\right)\left(\zeta_{2}-\zeta_{1}\right) \omega_{0}^{3}\right. \\
& +4 \zeta_{1}\left(C_{L} M_{L}^{-1}-4 p_{r}\right)\left(\zeta_{2}-\zeta_{1}\right) \omega_{0}^{2} \\
& +2\left(\zeta_{2}-\zeta_{1}\right)\left(M_{L}^{-1} K_{a}-M_{L}^{-2} C_{L}^{2}-4 M_{L}^{-1} C_{L} p_{r}-6 p_{r}^{2}\right) \omega_{0} \\
& +4 p_{r}^{3}+6 M_{L}^{-1} C_{L} p_{r}^{2}+4 M_{L}^{-2} C_{L}^{2} p_{r}-4 K_{a} M_{L}^{-1} p_{r} \\
& \left.+M_{L}^{-3} C_{L}^{3}-2 M_{L}^{-2} C_{L} K_{a}\right\} \\
& k_{r 4}=J_{M} M_{L} R_{b} K_{a}^{-1}\left\{8 \zeta_{1}\left(\zeta_{2}-\zeta_{1}\right)\left(2 \zeta_{1}^{2}-1\right) \omega_{0}^{4}\right. \\
& +8 p_{r}\left(\zeta_{2}-\zeta_{1}\right)\left(4 \zeta_{1}^{2}-1\right)+24 p_{r}^{2} \zeta_{1}\left(\zeta_{2}-\zeta_{1}\right) \omega_{0}^{2} \\
& \left.+8 p_{r}^{3}\left(\zeta_{2}-\zeta_{1}\right) \omega_{0}-p_{r}^{4}\right\} \\
& k_{r 5}=2 J_{M} M_{L} R_{b} K_{a}^{-1} \omega_{0}\left(\zeta_{2}-\zeta_{1}\right)\left\{\left(12 \zeta_{1}^{2}-16 \zeta_{1}^{4}-1\right) \omega_{0}^{4}\right. \\
& +16 \zeta_{1} p_{r}\left(1-2 \zeta_{1}^{2}\right) \omega_{0}^{3}+6 p_{r}^{2}\left(1-4 \zeta_{1}^{2}\right) \omega_{0}^{2} \\
& \left.-8 \zeta_{1} p_{r}^{3} \omega_{0}-p_{r}^{4}\right\} \\
& k_{r 6}=J_{M} M_{L} R_{b} K_{a}^{-1} \omega_{0}^{2}\left(\zeta_{2}-\zeta_{1}\right)\left\{\zeta_{1}\left(64 \zeta_{1}^{4}-64 \zeta_{1}^{2}+12\right) \omega_{0}^{4}\right. \\
& +8 p_{r}\left(16 \zeta_{1}^{4}-12 \zeta_{1}^{2}+1\right) \omega_{0}^{3}+48 \zeta_{1} p_{r}^{2}\left(2 \zeta_{1}^{2}-1\right) \omega_{0}^{2} \\
& \left.+8 p_{r}^{3}\left(4 \zeta_{1}^{2}-1\right) \omega_{0}+4 \zeta_{1} p_{r}^{4}\right\} \text {. }
\end{aligned}
$$

Figure 10 shows the estimation characteristics of FFRFO from $F_{L}$ to $\hat{F}_{\text {ext }}$. In the case of conventional multiple-root designed FFRFO, the gain characteristic increases in frequency band over the dither frequency $\omega_{0}$. On the other hand, the proposed notch-type FFRFO suppresses gain increase. 


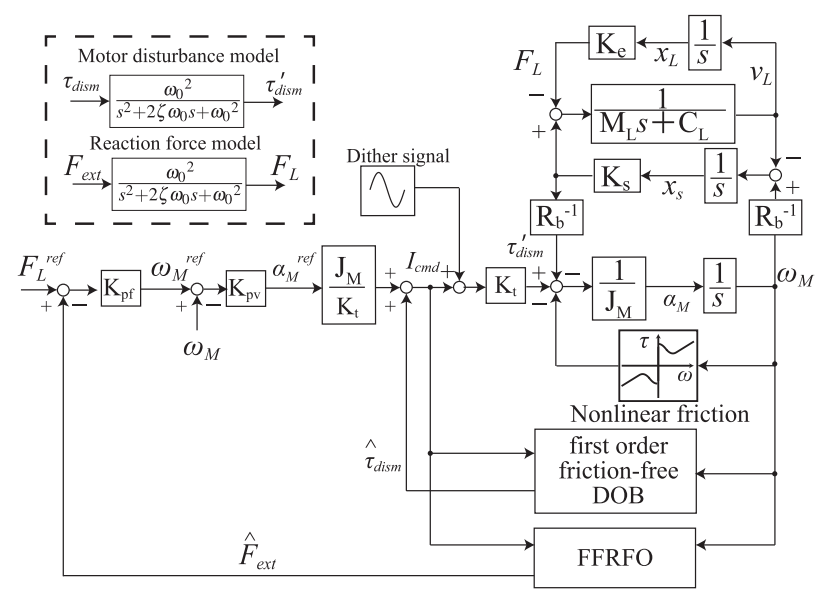

Fig. 11. Sensorless force control system

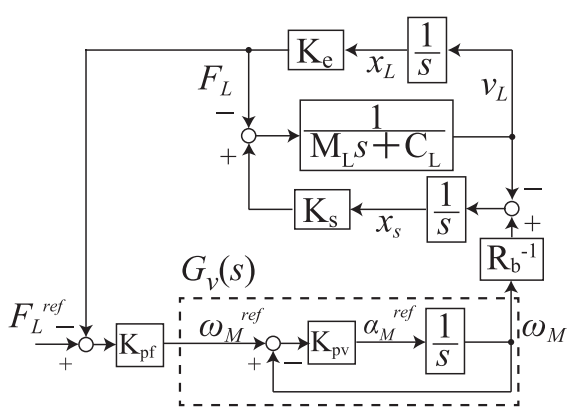

Fig. 12. Equivalent block of Fig. 11 (idealizing frictionfree DOB and FFRFO)

\section{Force Sensorless Force Control based on Ac- celeration Control}

Figure 11 shows the force sensorless force control system used in this paper. This system consists of the acceleration control system with first-order friction-free DOB, the proportional $(\mathrm{P})$ velocity controller and $\mathrm{P}$ force controller. In order to realize force sensorless control, the $\mathrm{FFRFO}^{(11)}$ is used to estimate the load-side reaction force.

The velocity control gain is designed under the assumption that the acceleration control loop is ideal, as shown in Fig. 12. In this case, the transfer function of the velocity control system $G_{v}(s)$ is expressed as follows:

$$
G_{v}(s)=\frac{\omega_{M}}{\omega_{M}^{r e f}}=\frac{K_{p v}}{s+K_{p v}} .
$$

When designed by the pole placement method, the velocity control gain is expressed as follows:

$$
K_{p v}=-p_{v}
$$

The transfer function, from the reaction force reference $F_{L}^{r e f}$ to the load-side reaction force $F_{L}$, is expressed as follows:

$$
\frac{F_{L}}{F_{L}{ }^{r e f}}=\frac{\frac{K_{s} K_{e}}{R_{b}} K_{p f} G_{v}(s)}{M_{L} s^{3}+C_{L} s^{2}+\left(K_{s}+K_{e}\right) s+\frac{K_{s} K_{e}}{R_{b}} K_{p f} G_{v}(s)} .
$$

In this case, the force control gain is designed by the try and error because the number of denominator orders exceeds the number of gains.
Table 1. Simulation conditions of acceleration control

\begin{tabular}{lc|l}
\hline Poles of friction-free disturbance observer & $p_{d}[\mathrm{rad} / \mathrm{s}]$ & -300 \\
Dither angler frequency & $\omega_{0}[\mathrm{rad} / \mathrm{s}]$ & $10 \pi, 90 \pi$ \\
\hline
\end{tabular}
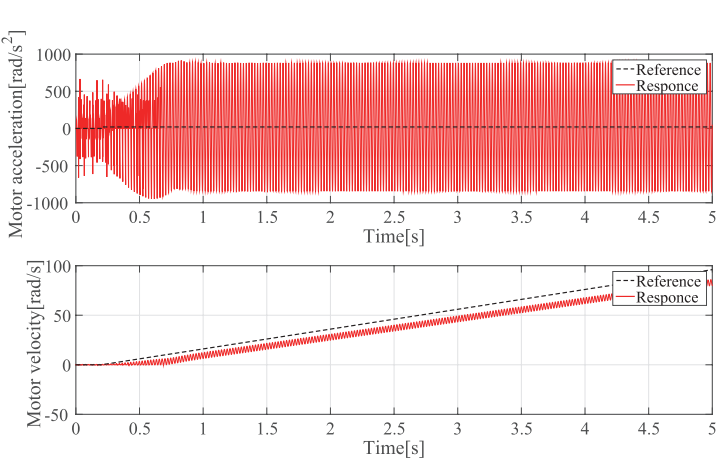

(a) Dither angler frequency: $\omega_{0}=90 \pi \mathrm{rad} / \mathrm{s}$
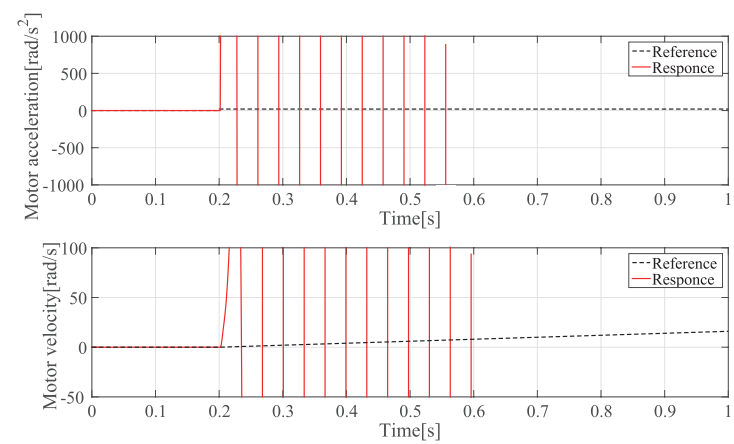

(b) Dither angler frequency: $\omega_{0}=10 \pi \mathrm{rad} / \mathrm{s}$

Fig. 13. Simulation results of zero-order friction-free DOB (conventional multiple roots design)

\section{Numerical Simulations}

In order to confirm the effectiveness of notch-type frictionfree DOB, numerical simulations of acceleration control are carried out. Table 1 shows the simulation conditions. The simulation model is the actual robot arm and environment shown in Fig. 1. The acceleration reference is a step signal of $20 \mathrm{~N}$. The velocity reference shows the integrated acceleration reference. The numerical simulation results are shown in Fig. 13-Fig. 16. Fig. 13(a), Fig. 14(a) and Fig. 16(a) show the accelaration response and speed response of motor side in the case of the dither frequency is set to $90 \pi \mathrm{rad} / \mathrm{s}$. From these resrults, the acceleration control systems by using all types of friction free DOB are able to control the suitable speed response. In case of the dither frequency is set to $10 \pi \mathrm{rad} / \mathrm{s}$, the speed responses by using multi-root designed frictionfree DOB are unstable in Figs. 13(b) and 14(b).

On the other hand, when using the proposed notch-type friction-free DOB, the velocity response follows the reference in Fig. 16.

Low-frequency makes the velocity vibration larger compared to the one in the high-frequency dither signal. Therefore, the low-frequency dither signal is more effective to static friction suppression.

\section{Experiments}

6.1 Experimental Setup The experimental system use the ball screw axis of an actual industrial robot as shown in Fig. 17. The robot pushes on the iron environment using 

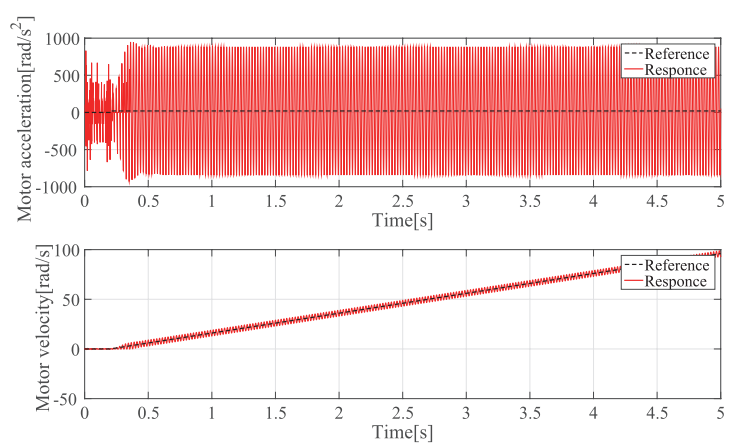

(a) Dither angler frequency: $\omega_{0}=90 \pi \mathrm{rad} / \mathrm{s}$
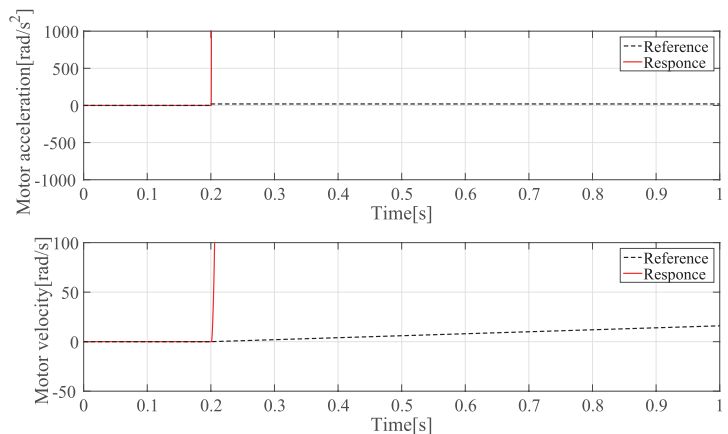

(b) Dither angler frequency: $\omega_{0}=10 \pi \mathrm{rad} / \mathrm{s}$

Fig. 14. Simulation results of first-order friction-free DOB (multiple roots design)
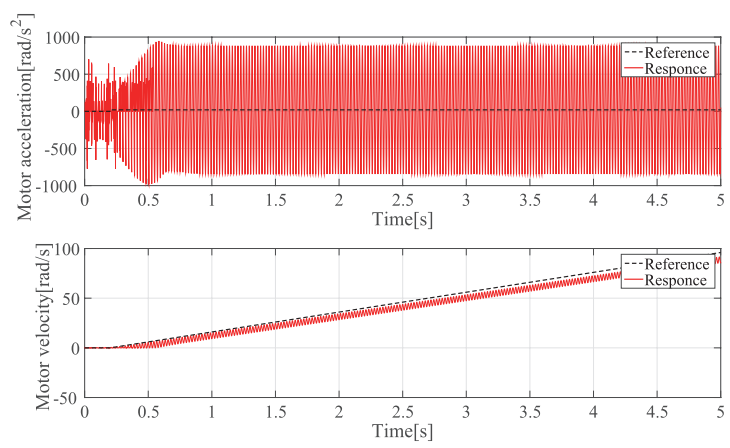

(a) Dither angler frequency: $\omega_{0}=90 \pi \mathrm{rad} / \mathrm{s}$
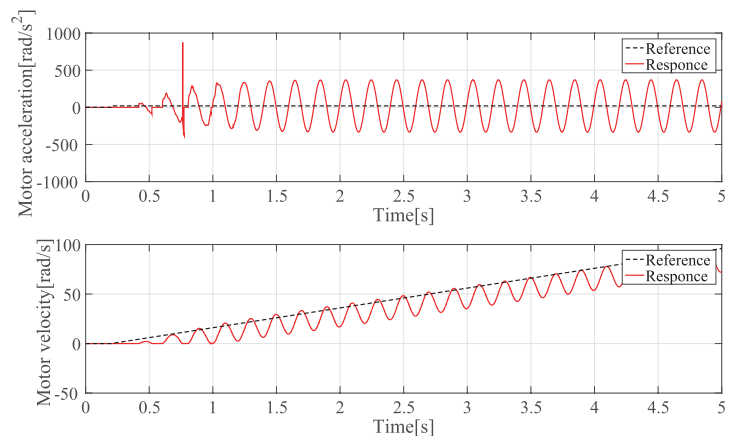

(b) Dither angler frequency: $\omega_{0}=10 \pi \mathrm{rad} / \mathrm{s}$

Fig. 15. Simulation results of zero-order friction-free DOB (proposed notch-type design)

the ball screw axis. The effectiveness of notch-type frictionfree DOBs is confirmed by using force sensorless force control system as shown in Fig. 11. Table 2 shows the experimental conditions of force sensorless force control. The dither frequency is set to the lowest possible frequency. The dither signal with lower frequency is more effective in suppressing static friction. The dither amplitude is $\pm 9 \%$ of the
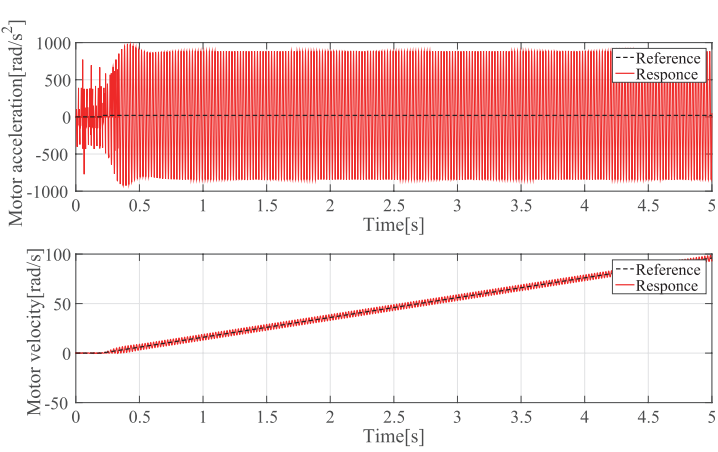

(a) Dither angler frequency: $\omega_{0}=90 \pi \mathrm{rad} / \mathrm{s}$
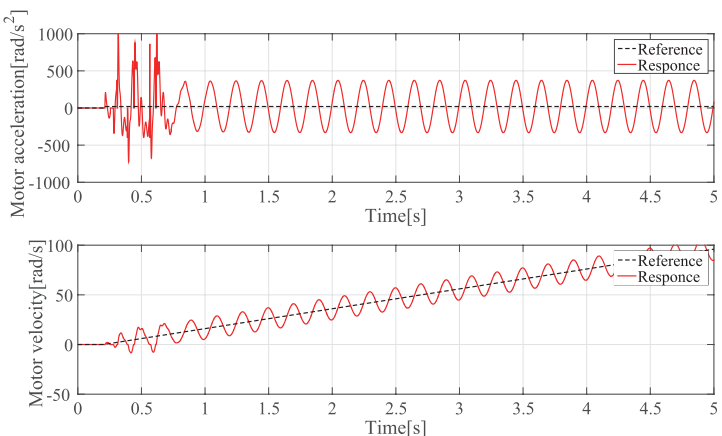

(b) Dither angler frequency: $\omega_{0}=10 \pi \mathrm{rad} / \mathrm{s}$

Fig. 16. Simulation results of first-order friction-free DOB (proposed notch-type design)

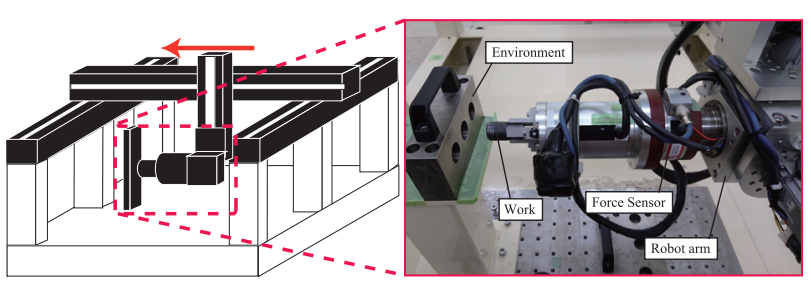

Fig. 17. Experimental equipments

Table 2. Setup parameters of force sensorless force control

\begin{tabular}{lc|l}
\hline Poles of friction-free disturbance observer & $p_{d}[\mathrm{rad} / \mathrm{s}]$ & -300 \\
Poles of FFRFO & $p_{r}[\mathrm{rad} / \mathrm{s}]$ & -250 \\
Poles of velocity controller & $p_{v}[\mathrm{rad} / \mathrm{s}]$ & -60 \\
Proportional gain of force controller & $K_{p f}$ & 0.006 \\
\hline
\end{tabular}

rated motor torque. The most suitable value determines by trial and error in this study, because the vibrational torque of $\pm 4.9 \mathrm{~N}$ does not impact the assembly application.

6.2 Zero-order Friction-free DOB Figure 18 shows the experimental results of force sensorless force control using zero-order friction-free DOB. The dither frequency of the conventional zero-order friction-free DOB is set to $25 \mathrm{~Hz}$. The dither frequency of the proposed notch-type friction-free DOB is set to $5 \mathrm{~Hz}$. Since the dither signal is set to low frequency, the estimation error decreases from $5.69 \mathrm{~N}$ to $4.74 \mathrm{~N}$ in $15 \mathrm{~s}$.

6.3 First-order Friction-free DOB Figure 19 shows the experimental results of force sensorless force control using first-order friction-free DOB. The dither frequency of the first-order friction-free DOB, designed with multiple roots, is set to $45 \mathrm{~Hz}$. The dither frequency of the proposed notch-type friction-free DOB is set to $25 \mathrm{~Hz}$. Thus, the transient response is faster when compared to using 


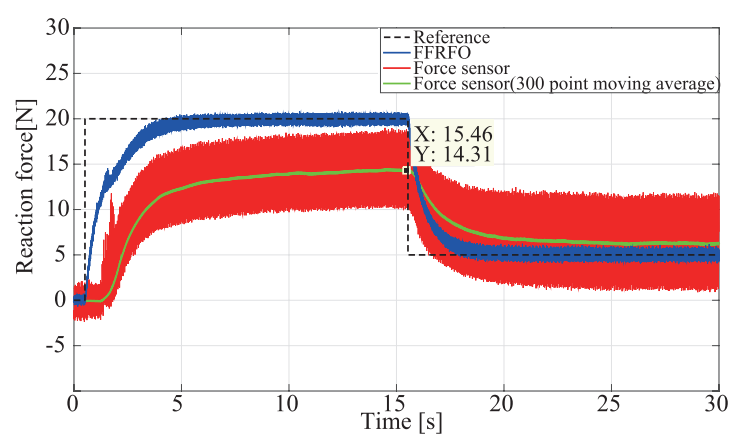

(a) Zero-order friction-free DOB (conventional multiple roots, $\omega_{0}=2 \pi 25 \mathrm{rad} / \mathrm{s}$ )

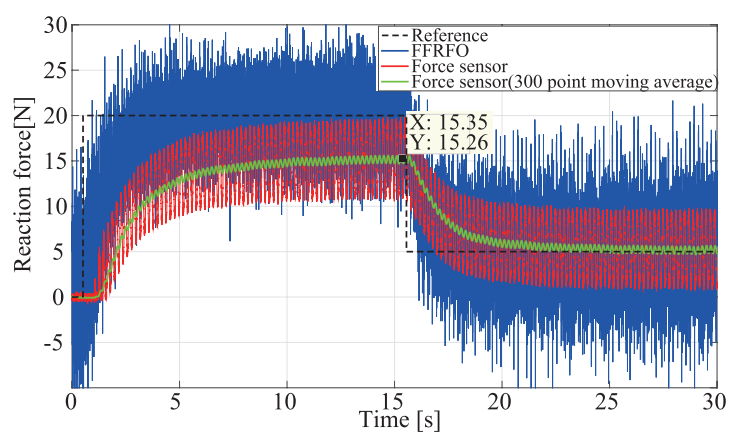

(b) Zero-order friction-free DOB (proposed notch-type, $\omega_{0}=2 \pi 5 \mathrm{rad} / \mathrm{s}$ )

Fig. 18. Experimental results of force sensorless force control using conventional and proposed zero-order friction-free DOB

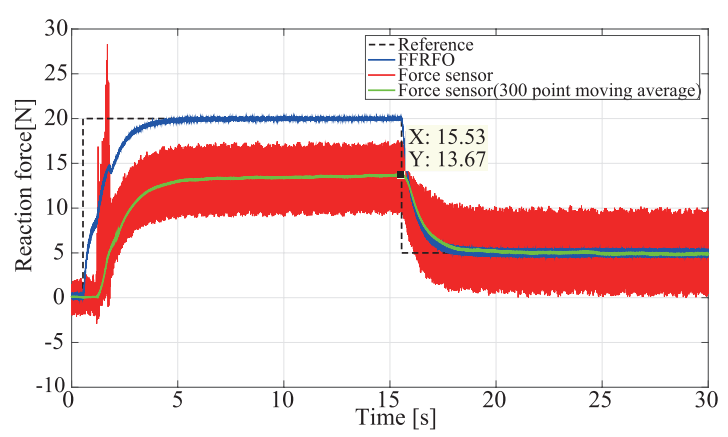

(a) First-order friction-free DOB (multiple roots, $\omega_{0}=2 \pi 45 \mathrm{rad} / \mathrm{s}$ )

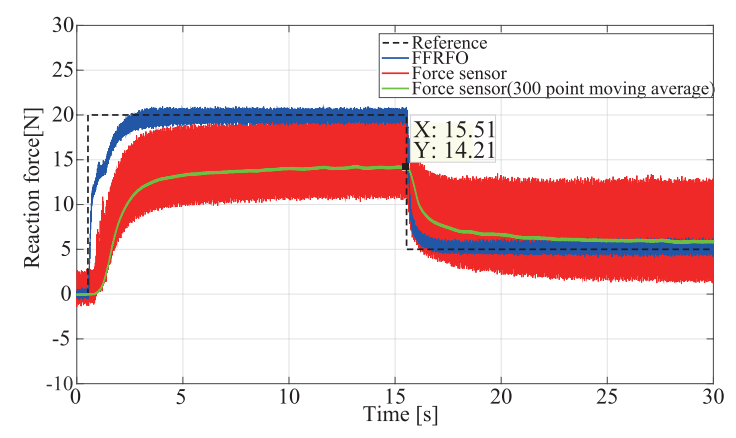

(b) First-order friction-free DOB (proposed notch-type, $\omega_{0}=2 \pi 25 \mathrm{rad} / \mathrm{s}$ )

Fig. 19. Experimental results of force sensorless force control using conventional and proposed first-order friction-free DOB

zero-order friction-free DOB. Similar to using zero-order friction-free DOB, the estimation error is decreased due to using notch-type friction-free DOB. However, the estimation error shown in Fig. 18(b) is smaller than the one shown in Fig. 19, since the dither frequency is different.

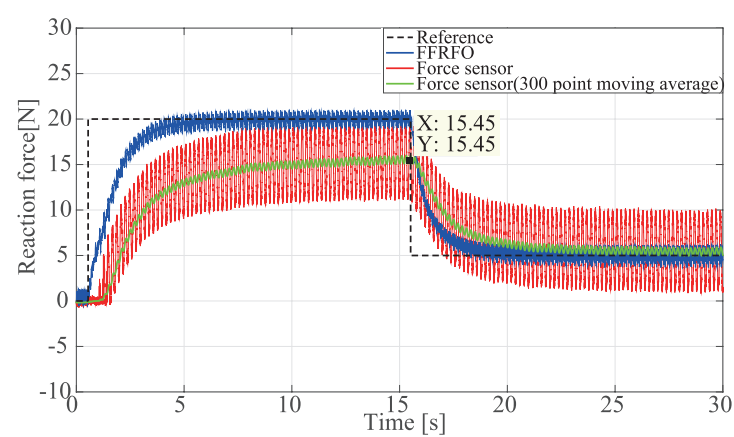

Fig. 20. Experimental results of force sensorless force control using proposed notch-type FFRFO and zero-order friction-free DOB (proposed notch-type design, $\omega_{0}=$ $2 \pi 5 \mathrm{rad} / \mathrm{s})$

6.4 Notch-type FFRFO Next, this paper confirms the validity of the notch-type FFRFO. Although Fig. 18 has smallest estimation error, the estimated force of FFRFO is too noisy. The poles of FFRFO are set to multiple roots. Therefor, the noise in frequency-band from dither signal frequency to observer poles is amplified. Because gain of estimation characteristic in frequency-band from dither signal frequency to observer poles is increased. Thus, this paper realizes FFRFO to notch-type poles same as the friction-free DOB. Fig. 20 shows the experimental results using notchtype FFRFO. The zero-order friction-free DOB is designed similar to what is shown in Fig. 18. The estimation noise of the notch-type FFRFO is decreased compared with conventional FFRFO.

\section{Conclusion}

In order to improve the force estimation performance, this paper proposes a new notch-type friction-free DOBs. In this paper, both friction-free DOB and FFRFO are realized as notch-type observers. As the result, the proposed force sensorless force control, based on the acceleration controller with the proposed notch-type friction-free DOB, realizes the fine force response that is less influenced by the nonlinear static friction. As a result, the dither signal frequency is set to lowest frequency and the force estimation error is decreased because static friction is suppressed. The effectiveness of the proposed system is confirmed by the experiments using an actual industrial robot.

\section{References}

( 1 ) K. Kiguchi and T. Fukuda: "Position/Force Control of Robot Manipulators for Geometrically Unknown Objects Using Fuzzy Neural Networks", IEEE Transactions on Industrial Electronics, Vol.47, No.3, pp.641-649 (2000)

( 2 ) S.J. Huang and C.Y. Shy: "Fuzzy logic for constant force control of end milling", IEEE Trans. Robotics and Automation, Vol.46, No.1, pp.169-176 (1999)

( 3 ) T. Insperger, L.L. Kovacs, P. Galambos, and G. Stepan: "Increasing the Accuracy of Digital Force Control Process Using the Act-and-Wait Concept", IEEE/ASME Trans. on Mechatronics, Vol.15, No.2, pp.291-298 (2009)

( 4 ) K. Kaneko, K. Tanie, and K. Komoriya: "Torque Control of a DC Motor with Reduction Gears (3rd Report, Drive Control System with Flexible Joint and Its Torque Control Characteristics)", Trans. of the Japan Society of Mechanical Engineers (C), Vol.62, No.602, pp.133-140 (1996)

( 5 ) M. Hashimoto, Y. Kiyosawa, and R.P. Paul: "A torque sensing technique for robots with harmonic drives", IEEE Trans. Robotics and Automation, Vol.9, No.1, pp.108-116 (1993) 
( 6 ) I. Godler, M. Horiuchi, M. Hashimoto, and T. Ninomiya: "Accuracy Improvement of Built-In Torque Sensing for Harmonic Drives", IEEE/ASME Transactions on Mechatronics, Vol.5, No.4, pp.360-366 (2000)

( 7 ) M. Hashimoto, T. Hattori, M. Horiuchi, and T. Kamata: "Development of a Torque Sensing Robot Arm for Interactive Communication", Proceedings of the IEEE International Workshop on Robot and Human Interactive Communication, ROMAN2002-BERLIN, pp.344-349 (2002)

( 8 ) Y. Kuroki, Y. Kosaka, T. Takahashi, E. Niwa, H. Kaminaga, and Y. Nakamura: "Cr-N Alloy Thin-Film Based Torque Sensors and Joint Torque Servo Systems for Compliant Robot Control", Proceedings of the IEEE International Conference on Robotics and Automation 2013, ICRA2013KARLSRUHE, pp.4939-4943 (2013)

( 9 ) T. Murakami, F. Yu, and K. Ohnishi: "Torque sensor-less Control in Multidegree-of-Freedom Manipulator", IEEE Trans. on Industrial Electronics, Vol.40, No.2, pp.259-265 (1993)

(10) Y. Ohba, M. Sazawa, K. Ohishi, T.Asai, K. Majima, Y. Yoshizawa, and K. Kageyama: "Sensorless Force Control for Injection Molding Machine Using Reaction Torque Observer Considering Tortion Phenomenon", IEEE Trans. on Industrial Electronics, Vol.56, No.8, pp.2955-2960 (2009)

(11) K. Ohishi and R. Furusawa: "Fine Actuator Force Control for Electric Injection Molding Machines", IEEE Industrial Electronics Magazine, pp.4-13 (2012)

(12) C. Mitsantisuk, M. Nandayapa, K. Ohishi, and S. Katsura: "Design for Sensorless Force Control of Flexible Robot by Using Resonance Ratio Control Based on Coefficient Diagram Method", AUTOMATIKA, 54-1, pp.62-73 (2013)

(13) T.T. Phuong, K. Ohishi, Y. Yokokura, and C. Mitsantisuk: "FPGA-Based High-Performance Force Control System With Friction-Free and Noise-Free Force Observation", IEEE Trans. on Industrial Electronics, Vol.61, No.2, pp.994-1008 (2014)

(14) K. Yuki, T. Murakami, and K. Ohnishi: "Vibration Control of a 2 Mass Resonant System by the Resonance Ratio Control", IEEJ Trans. on Ind. Appli., Vol.113, No.10, pp.1162-1169 (1993)

(15) Y. Hori: "2-Inertia System Control using Resonance Ratio Control and Manabe Polynomials", IEEJ Trans. Ind. Appli., Vol.114, No.10, pp.1038-1045 (1994)

(16) S. Morimoto, H. Kameyama, and Y. Takeda: "Comparison of Resonance Ratio Control and $H^{\infty}$ Control on Speed Control of Two-mass System", IEEJ Trans. on Ind. Appli., Vol.116, No.6, pp.678-684 (1996)

(17) S. Katsura and K. Ohnishi: "Force Servoing by Flexible Manipulator Based on Resonance Ratio Control”, IEEE Trans. on Industrial Electronics, Vol.54, No.1, pp.539-547 (2007)

(18) Y. Yokokura and K. Ohishi: "Robust Stability Analysis of Load-side Inertia Variation in Torsion Torque Control with a Resonance Ratio Controller", Proceedings of the IEEJ International Workshop on Sensing, Actuation, Motion Control, and Optimization, SAMCON2017-NAGAOKA, pp.1-4 (2017)

\section{Appendix}

\section{Steady State Error}

We think that this steady state error of $4.9 \mathrm{~N}$ is caused by static friction. To overcome this problem, the dither signal is added to the torque reference. Then, the proposed system has little influence on static friction. To confirm the influence of static friction, we carry out a simulation of the force sensorless force control, including the nonlinear friction model. app. Fig. 1 shows the simulation block diagram.

Numerical simulation results are shown in app. Fig. 2. In app. Fig. 2(a), the steady state error of the control system without the static friction model is zero. On the other hand, in app. Fig. 2(b), the steady state error of the control system with the static friction model is non-zero.

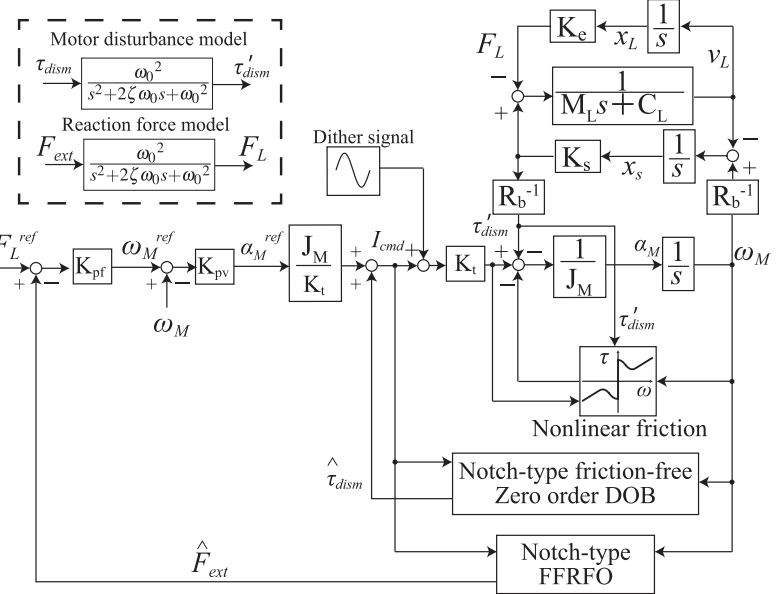

app. Fig. 1. The force sensorless force control including the nonlinear friction

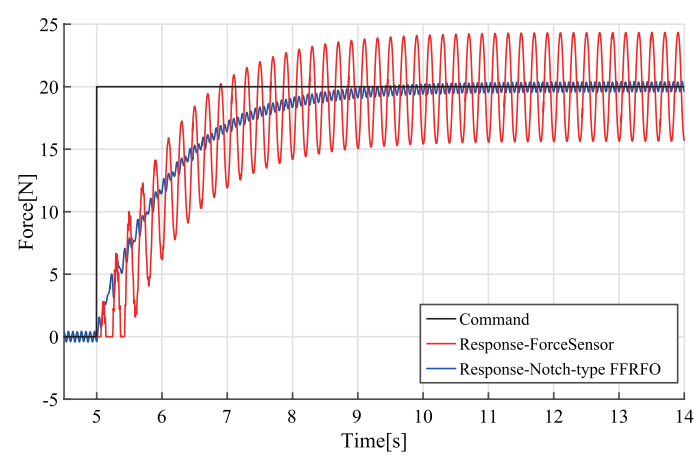

(a) Without static friction model

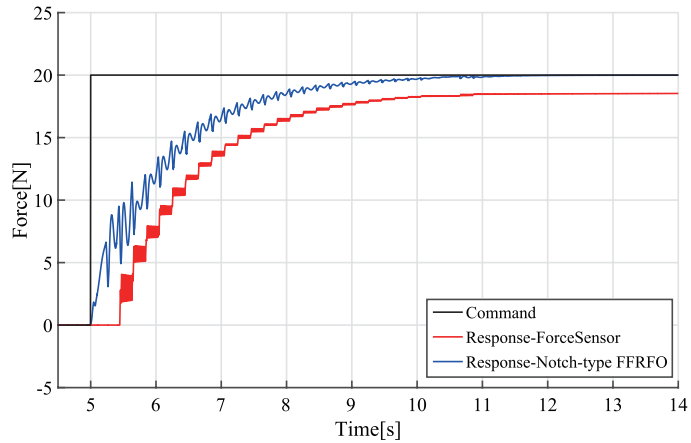

(b) Within static friction model

app. Fig. 2. Numerical simulation result of force sensorless force control (Dither Signal: sin wave, Frequency: $5 \mathrm{~Hz}$, Amplitude: $\pm 9 \%$ )
Hiroshi Nakamura (Student Member) received B.E. degrees in elec-

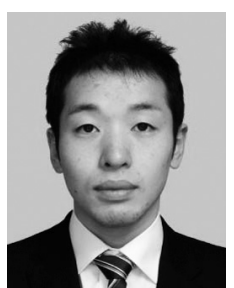
rical engineering from Nagaoka University of Technology, Nagaoka, Japan, in 2015. Now he is a candidate of the M.E. degree in Electrical, Electronics and Information Engineering. His research interests include motion control. He is a member of the Institute of Electrical Engineering. 
Kiyoshi Ohishi (Fellow) received the B.E., M.E., and Ph.D. degrees

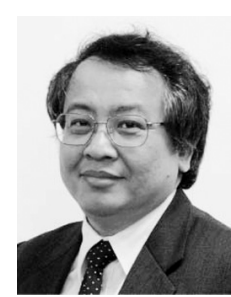
in electrical engineering from Keio University, Yokohama, Japan, in 1981, 1983, and 1986, respectively. From 1986 to 1993, Prof. Ohishi was an Associate Professor with Osaka Institute of Technology, Osaka, Japan. Since 1993, he has been with Nagaoka University of Technology, Nagaoka, Japan. He became a Professor in 2003. His research interests include motion control, mechatronics, robotics and. power electronics. He is a General chair of IEEE IECON2015. He is a General chair of IEEE AMC2010 and AMC2016. He also contributes as an AdCom Member of IEEE/IES and an Associate Editor for the IEEE Transactions on Industrial Electronics. He is a member of the Institute of Electrical Engineering of Japan and the Robotics Society of Japan. He received the Outstanding Paper Awards at IECON'85 and Best Paper Awards at IECON'02, IECON'04 from the IEEE Industrial Electronics Society.

Yuki Yokokura (Member) received the B.E. and M.E. degrees in elec-

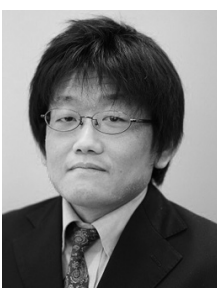
trical engineering from Nagaoka University of Technology, Niigata, Japan, in 2007 and 2009, respectively. In 2011, he received Ph.D. degree in integrated design engineering from Keio University, Yokohama, Japan. From 2010 to 2011, he was a JSPS (Japan Society for the Promotion of Science) Research Fellow (DC2 and PD). He was a Visiting Fellow at Keio University, and a Postdoctoral Fellow at Nagaoka University of Technology in 2011. Since 2012, he has been an Assistant Professor with Nagaoka University of Technology. His research interests include motion control, motor drive, powerelectronics, and real-world haptics.

Naoki Kamiya

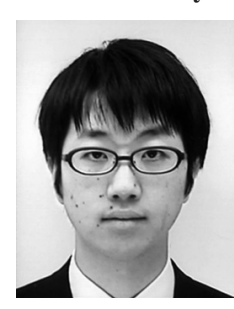

Student Member) received B.E. degree in Electrical, Electronics and Information Engineering from Nagaoka University of Technology, Japan in 2017. Now he is a candidate of the M.E. degree in Electrical, Electronics and Information Engineering. His research interests include motion control. He is a student member of the Institute of Electrical Engineers of Japan (IEEJ).
Toshimasa Miyazaki (Member) received the B.E., M.E., and Ph.D.

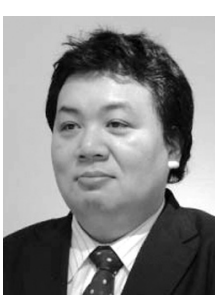
degrees all in electrical engineering, from Nagaoka University of Technology, Niigata, Japan, in 1994, 1996, and 1999, respectively. From 1999 to 2009, he was an Associate Professor with Nagaoka National College of Technology, Niigata, Japan. Since 2010, he has been an Associate Professor with Nagaoka University of Technology, Niigata, Japan. His research interests include motion control and power electronics. He is a member of the IEEE Industrial Electronics Society (IEEE IES), the Institute of Electrical Engineers of Japan (IEEJ), and the Society of Instrument and Control Engineers (SICE).

Akifumi Tsukamoto (Member) received the B.E. and M.E. degrees

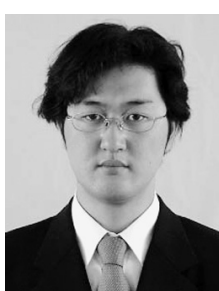
in electronics engineering from Sojo University, $\mathrm{Ku}-$ mamoto, Japan, in 2005 and 2007. He is currently with Hirata Co.,Ltd., Kumamoto, Japan. He is involved in the development of industrial robot controllers. 